\title{
Performance Analysis of Device-to-Device Communication Underlaying Dense Networks (DenseNets)
}

\author{
Shruti Gupta ${ }^{1}$, Muralikrishnan Srinivasan ${ }^{2},{\text { Yan } \operatorname{Lin}^{3}, \text { R. Zhang }}^{1}$, S. Kalyani ${ }^{2}$ and L. Hanzo ${ }^{1}$
}

\begin{abstract}
Given the exponential proliferation of mobile devices and broadband services, there is a pressing need to amalgamate multiple technologies accommodating infrastructural changes for meeting the ever-increasing demands of user capacity. Therefore we propose the integration of proximitybased direct device-to-device (D2D) communication with the network densification concept. In this treatise, we consider user-centric non-overlapping clusters associated with the closest access point (AP) for serving user equipment (UE) in the downlink under limited availability of orthogonal resource blocks (RBs), while the D2D links also reuse these downlink RBs. We analytically derive the coverage probability as well as the average transmission rate of $\mathrm{D} 2 \mathrm{D}$ links under the proposed model, which results in a complex interference scenario. The performance results verify that our analytical results closely match with simulations for different parametric settings. The impact of the presence of $D 2 D$ links on the coverage probability of the UEs is also quantified, revealing that the UEs' performance is only modestly affected under the proposed system model.
\end{abstract}

\section{INTRODUCTION}

In order to meet the continuing upsurge of data traffic spurred by the proliferation of wireless services, future wireless networks should support an increased capacity, whilst improving the energy efficiency, cost and spectrum utilization. Hence, future cellular wireless networks are expected to rely on a mixture of different technologies whilst readily accommodating infrastructural changes for supporting the escalating tele-traffic demands. The different technologies to be accommodated include, but are not limited to deviceto-device (D2D) communication, massive machine communication, ultra-reliable communication, network densification [1]-[3] etc. The integration of D2D communication and the concept of network densification would present a new paradigm shift for future networks with the aim of increasing the capacity and energy efficiency of radio links as well as enabling a better exploitation of the under-utilized spectrum.

On one hand, D2D communication enables a pair of closely located mobile users to establish a direct link for

1. School of Electronics and Computer Science, University of Southampton, SO17 1BJ, United Kingdom.

Corresponding Author's Email: lh@ecs.soton.ac.uk

2. Electrical Department, Indian Institute of Technology, Madras

3. School of Information Science and Engineering, Southeast University, Nanjing, China

L. Hanzo would like to acknowledge the financial support of the Engineering and Physical Sciences Research Council projects EP/Noo4558/1, EP/PO34284/1, COALESCE, of the Royal Society's Global Challenges Research Fund Grant as well as of the European Research Council's Advanced Fellow Grant QuantCom.

S. Gupta and M. Srinivasan are co-first authors. their user plane traffic without going through the entire network infrastructure, while reusing the spectrum allocated for traditional cellular communication, thereby offloading the backhaul traffic and enhancing both the energy- and spectralefficiency [4], [5]. However, D2D communication underlaying cellular networks will bring about many new challenges, including sophisticated interference management due to the co-existence of different traffic patterns, different spectral bands and diverse user densities in the network etc. Hence, recent research activities have been devoted to investigating the potential of D2D-based cellular networks [6]-[9].

The seminal studies of [10]-[15] were focused on the performance of D2D communication underlaying cellular networks, where the Poisson point process (PPP) was used for modelling the spatial distribution of the D2D users and base stations (BSs). Further advances were made by the authors of [4], [5], [7], [8], who considered a single-tier network where a single base station (BS) transmits data to the UEs in the downlink associated with a D2D underlay. However, in the latter contributions the distribution of D2D users was not modelled by a PPP.

On the other hand, network densification [16], [17] is a combination of spatial densification (increasing number of antennas per node and access points/user density) and spectral aggregation resulting in improving spatial reuse as well as network capacity. Since the network densification introduces changes in the definition of networks in liu of density of APs, users and spectral resources, there are bound to be impact of this over the communication and thus has been analysed in literature [18]-[24].

Explicitly, Sheng et al. in [18], investigated the influence of network densification on the coverage probability (CP) and area spectral efficiency (ASE) of the downlink cellular network. Contrastingly, authors of [20] and [24] considered the practical path loss model incorporating both line-ofsight (LoS) and non-line-of-sight (NLoS) transmissions for analysing their impact on CP and ASE of the downlink and uplink of dense small cell networks (SCNs), respectively. Furthermore, in [22], authors incorporated channel models relying on LoS/NLoS propagation as well as using both bounded path loss model and the dominant BS based approach for deriving the conditional distributions of intercell interference in the dense network. Shojaeifard et al. [19] used stochastic geometry theory for developing an analytical framework for identifying the most energy efficient deployment framework meeting certain minimum service 
criteria where load-awareness was explicitly embedded in the dense cellular network design. On the contrary, considering user centric adaptive clustering approach authors in [21] investigated the performance of coordinated multi-point transmissions in dense cellular network. The impact of BS idle mode capacity on the performance of dense multi-tier heterogeneous network was analysed in [23] when the BS density exceeds the UE density.

Against this background, we consider the downlink of a two-tier hybrid dense network (DenseNet) consisting of densified APs supporting multiple UEs that are associated with the closest AP and underlay D2D links which reuse the spectral resources of the DenseNet. Since we consider limited spectrum availability for downlink transmissions in a DenseNet, in order to serve the UEs, the spectrum is reused by the APs to support all the UEs as well as by the D2D links for their direct communication. This integration would present a sophisticated system, where the UEs would suffer from the interference imposed by D2D communication as well as by the other UEs served in co-channel bands and vice versa. There is a paucity of research on DenseNet incorporating underlaid D2D links except the work in [25]. Poulakis et.al. [25] investigated the impact of network densification on D2D communication in the context of radio resource management of multi-cell system. However, it carried out the system level simulations for uplink throughput without representing any analytical expression.

In contrast to [10]-[15], in this treatise, the spatial distribution of the nodes is not based on the idealized PPP model, when studying the effect of the number of APs/UEs/D2D links on the system's performance. Instead, we found that at the time of writing there are no coverage probability/rate expressions for our Control/User plane separation based architecture. Motivated by this open problem in the literatur, we have embarked on investigating the D2D enabled DenseNet concept by analysing both the coverage as well as the rate of D2D links. Our expressions are compact, requiring the numerical evaluation of simple mathematical functions and can be directly used for evaluating the coverage probability versus the number of APs/UEs/D2Ds or the number of resource blocks (RBs).

The rest of the paper is organized as follows. In Section II, our hybrid system model is presented, followed by the derivation of coverage, approximate coverage which significantly simplifies the complexity of the expressions and average transmission rate of the D2D links in Section III. Our quantitative analysis based on the approximate coverage and discussions are presented in Section IV. Finally, we conclude in Section V.

\section{Network Model}

We consider a downlink two-tier hybrid DenseNet that consists of one macro BS (MBS) and densified multiantenna APs that support multiple UEs as well as D2D links. These single-antenna D2D links reuse the downlink

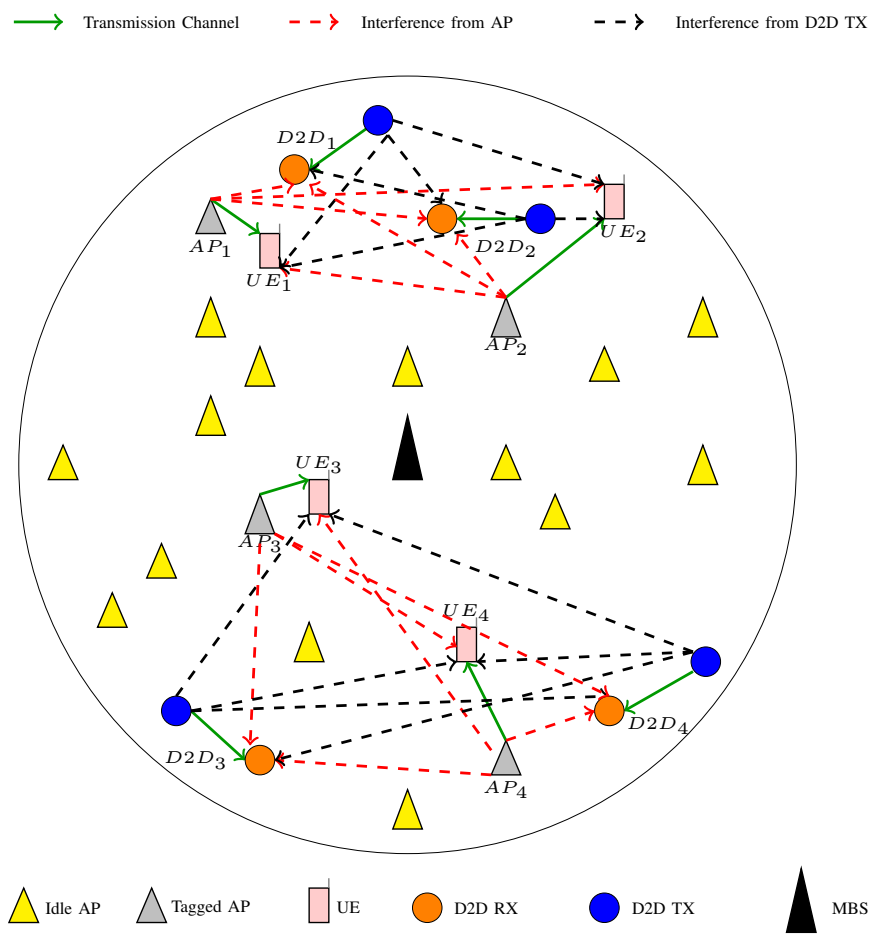

Figure 1. An illustration of the relevant transmission and interference patterns in our hybrid DenseNet, which is supporting $D=4$ D2D links and $K=4$ UEs, each of which associate with the best AP resulting in 4 tagged APs along with 14 idle APs from a total $L=18$ APs. The D2D links and UEs reuse $N=2$ orthogonal RBs, such that each RB is reused by 2 D2D links as well as 2 UEs, hence these D2D links imposes interference on the UEs communicating within the same RB as well as to other D2D links operating under co-channel and vice versa, as depicted in the figure.

spectrum of UEs for their communication. For the sake of providing an improved data rate and service control, we adopt the Control/User (C/U) split architecture of [26]. In this architecture, extra APs are deployed in hotspot areas for providing the user plane and the MBS provides the network control plane for the cell. The MBS controls the UE handovers, the management of data transmission and the resource reuse by D2D links, while the APs are responsible transmitting data to UEs in the downlink. Thus, we consider only co-tier interference in our system, while ignoring the cross-tier interference. We assume that all APs, UEs and D2D links are uniformly distributed across a cell having a radius of $R_{c}$ and that the density of APs is comparable to or possibly higher than that of UEs, while the density of D2D links is comparable to that of UEs.

Since we consider a user-centric architecture, the MBS assists each UE to form a non-overlapping cluster by selecting the best AP, which employs Maximum Ratio Transmission (MRT) beam-forming, as indicated in Fig. 1. As a result of densely distributed APs, each UE is associated with its closest AP, termed as tagged AP. Each AP would either serve a UE in the DL or it is assumed to be inactive, if it is not tagged with any UE. All APs share the same spectrum, which is 
partitioned into multiple orthogonal resource blocks (RBs). Considering that the orthogonal RBs are scarce relative to the density of UEs in a DenseNet, we assume that each RB can be allocated to multiple clusters, but each cluster is served by one RB. As seen in Fig. 1, $N=2$ orthogonal resource blocks are allocated to clusters serving UEs marked with $U E_{1}$ and $U E_{3}$, while clusters for $U E_{2}$ and $U E_{4}$ reuse these RBs. Since we consider the density of D2D links to be similar to that of the UEs, the RB reuse would follow a similar pattern, where each $\mathrm{RB}$ is reused by multiple D2D links, while each D2D link can reuse at most one RB. Again, as shown in Fig. 1, each RB is reused by 2 out of $D=4 \mathrm{D} 2 \mathrm{D}$ links. We assume a random allocation of $\mathrm{RBs}$ to UEs as well as for the $\mathrm{RB}$ reuse by $\mathrm{D} 2 \mathrm{D}$ links throughout this treatise.

An illustration of the relevant transmission and interference patterns in our user centric DenseNet with underlaid D2D links is given in Fig. 1. Each tagged AP creates interference for the UEs apart from its associated UE as well as for the D2D links that are reusing the same RB. On the other hand, each D2D transmission imposes interference on the DL communication of UEs and other D2D links that are transmitting in a co-channel RB, as clearly shown in Fig. 1. Let us define the network as follows:

\begin{tabular}{|c|c|}
\hline Symbol & Description \\
\hline$I_{k}$ & $\begin{array}{l}\text { set of interfering UEs, which uses the same } \\
\text { RB as user } k\end{array}$ \\
\hline$\phi_{k}$ & $\begin{array}{l}\text { set of interfering D2D links, which uses the } \\
\text { same RB as user } k\end{array}$ \\
\hline$\psi_{d}$ & $\begin{array}{l}\text { set of interfering UEs, which uses the same } \\
\text { RB as D2D } d\end{array}$ \\
\hline$\varphi_{d}$ & $\begin{array}{l}\text { set of interfering D2D links, which uses the } \\
\text { same RB as D2D } d\end{array}$ \\
\hline$p_{d}$ & transmit power of D2D link \\
\hline$p_{k}$ & transmit power of AP $k$ \\
\hline$h_{k, i, n}^{j}$ & $\begin{array}{l}\text { channel coefficient from the } k^{t h} \mathrm{AP} \text { to } \mathrm{UE} i \\
\text { on RB } n \text { from } j^{\text {th }} \text { antenna }\end{array}$ \\
\hline$h_{k, d, n}^{j}$ & $\begin{array}{l}\text { channel coefficient from the } k^{t h} \text { AP to } d^{t h} \\
\text { D2D RX on RB } n \text { from } j^{t h} \text { antenna }\end{array}$ \\
\hline$w_{k, n}^{j}$ & $\begin{array}{l}\text { beam-forming coefficient used by AP } k \text { to UE } \\
k \text { on RB } n \text { from } j^{t h} \text { antenna }\end{array}$ \\
\hline$g_{d, d, n}$ & $\begin{array}{l}\text { channel coefficient between the } d^{t h} \text { D2D TX- } \\
\text { RX link on RB } n\end{array}$ \\
\hline$g_{i, d, n}$ & $\begin{array}{l}\text { channel coefficient from } i^{t h} \text { D2D TX to RX } \\
\text { of D2D link } d \text { on RB } n\end{array}$ \\
\hline$g_{i, k, n}$ & $\begin{array}{l}\text { channel coefficient from } i^{t h} \text { D2D TX to } k^{t h} \\
\text { UE on RB } n\end{array}$ \\
\hline$M$ & the number of antennas at AP \\
\hline$L$ & number of APs \\
\hline$K$ & number of UEs \\
\hline$D$ & number of D2D links \\
\hline$N$ & number of RBs \\
\hline
\end{tabular}

SYMBOLS USED FOR NETWORK PARAMETERS.

The D2D communication is incorporated as a complement to the underlying DL communication and thus the UEs generally have a higher priority than the D2D links in a cell. The APs maintain reliable connection with the UEs under the power budget of $p_{c}^{\max }$, while the D2D link reuses the randomly matched $\mathrm{RB}$ of the UE under the power budget $p_{d}^{\max }$. We assume that the D2D TX is at a distance $x$ from the
D2D RX, where $x$ varies uniformly in $\left[R_{1}, R_{2}\right]$. All possible transmission channels in the network are considered to be independent and identically Rayleigh distributed throughout this treatise. Since the system is interference limited, we define the SIR of the $d^{\text {th }}$ D2D link as:

$$
\beta_{d, n}=\frac{p_{d}\left|g_{d, d, n}\right|^{2} x^{-\alpha}}{I_{u d}+I_{d}}
$$

where $\quad I_{d}=\sum_{i \in \varphi_{d}} p_{d}\left|g_{i, d, n}\right|^{2} r_{i, d}^{-\alpha} \quad$ and $\quad I_{u d}=$ $\sum_{k \in \psi,} \frac{p_{k}}{M} \sum_{j=1}^{M}\left|h_{k, d, n}^{j} w_{k, n}^{j}\right|^{2} l_{k, d}^{-\alpha} \quad$ represent the interference arriving from other D2D links and DL transmission of UEs respectively, that are served on the same RB. Furthermore, $p_{d}$ and $p_{k}$ represent the transmit powers of D2D links and that of the $k^{t h} \mathrm{AP}$, which is set equal to their power budgets of $p_{d}^{\max }$ and $p_{c}^{\max }$, respectively. The distance between the $d^{\text {th }}$ D2D pair is denoted by $x$, while $r_{i, d}$ and $l_{k, d}$ are the distances from the $i^{t h} \mathrm{D} 2 \mathrm{D} \mathrm{TX}$ and $k^{t h} \mathrm{AP}$ to $d^{t h} \mathrm{D} 2 \mathrm{D} \mathrm{RX}$, respectively. The $j^{\text {th }}$ component of the beam-forming vector is $w_{k, n}^{j}=\frac{h *_{k, k, n}^{j}}{\left|\vec{h}_{k, k, n}^{j}\right|}$.

Similarly, we define the SIR of the $k^{t h}$ UE served on the $n^{\text {th }} \mathrm{RB}$ as:

$$
\beta_{k, n}^{U E}=\frac{\frac{p_{k}}{M} \sum_{j=1}^{M}\left|h_{k, k, n}^{j} w_{k, n}^{j}\right|^{2} l_{k}^{-\alpha}}{I_{d u}+I_{u}}
$$

where $I_{u}=\sum_{i \in I_{k}} \frac{p_{i}}{M} \sum_{j=1}^{M}\left|h_{i, k, n}^{j} w_{i, n}^{j}\right|^{2} l_{i, k}^{-\alpha} \quad$ and $\quad I_{d u}=$ $\sum_{i \in \phi_{k}} p_{d}\left|g_{i, k, n}\right|^{2} r_{i, k}^{-\alpha}$ represent the interference imposed by the DL transmission of other UEs and D2D links respectively, that are served on the same RB. The variables $r_{i, k}$ and $l_{i, k}$ represent the distance from the $i^{t h} \mathrm{D} 2 \mathrm{D}$ TX and the $i^{t h} \mathrm{AP}$ to $k^{t h} \mathrm{UE}$, respectively.

\section{Coverage And RATE OF D2D Links}

In this section, we will first derive the coverage probability of D2D links, followed by the rate analysis of D2D links.

\section{A. Coverage Probability}

The probability of successful communication between the source and destination is defined as the coverage probability. In other words, the probability of the D2D links possessing an SIR higher than the target SIR (T) is known as the coverage probability of D2D links. It is affected by the distance $x$ between the D2D TX and the D2D RX, by the transmit power $p_{d}$ of the D2D link as well as by the interferences $I_{u d}$ and $I_{d}$ experienced at the D2D Rx due to the DL communication of UEs and the D2D communication operating on the same $\mathrm{RB}$, respectively. Let us now derive the coverage probability of the D2D links.

Theorem 1. The coverage probability of a typical D2D link can be formulated as Eq. (4),

$C P=\int_{0}^{2 R_{c}} \int_{0}^{2 R_{c}} \int_{R_{1}}^{R_{2}} P\left[\beta_{d, n}>T\right] f_{X}(x) d x f_{L}\left(l_{k, d}\right) d l_{k, d} f_{R}\left(r_{i, d}\right) d r(;, j)$ 


$$
\begin{gathered}
=\int_{R_{1}}^{R_{2}}\left[\int_{0}^{2 R_{c}}\left(\frac{1}{1+\frac{T p_{k} l_{k, d}^{-\alpha}}{M p_{d} x^{-\alpha}}}\right)^{M} f_{L}\left(l_{k, d}\right) d l_{k, d}\right]^{\frac{K}{N}-1} \times \\
{\left[\int_{0}^{2 R_{c}} \frac{1}{1+\frac{T p_{d} r_{i, d}^{-\alpha}}{p_{d} x^{-\alpha}}} f_{R}\left(r_{i, d}\right) d r_{i, d}\right]^{\frac{D}{N}-1} \frac{1}{R_{2}-R_{1}} d x}
\end{gathered}
$$

where $f_{X}(x), f_{R}\left(r_{i, d}\right)$ and $f_{L}\left(l_{k, d}\right)$ denote the probability density function of $x, r_{i, d}$ and $l_{k, d}$ respectively. Also, $\frac{D}{N}$ and $\frac{K}{N}$ are reuse factors of the D2D links and AP-UE links. ${ }^{1}$ In this treatise we assume that the distance $x$ between the $D 2 D$ $T X$ and $R X$ pair is uniformly distributed in $\left[R_{1}, R_{2}\right]$, thus we have $f_{X}(x)=\frac{1}{R_{2}-R_{1}}$. On the other hand, the $d^{\text {th }} D 2 D$ pair is located at a distance $l_{k, d}$ from the interfering APs and at a distance $r_{i, d}$ from the other interfering $D 2 D$ TXs. Since the APs and D2D RXs are uniformly distributed in a cell of radius $R_{c}$, while the D2D TXs are located at a distance $x$ from their respective $R X s, l_{k, d}$ is the distance between two uniformly located points, while $r_{i, d}$ is approximated as the distance between two uniformly located points, because we have $d<<R_{c}$. Hence their distribution can be written as [27]:

$$
\begin{aligned}
& f_{L}\left(l_{k, d}\right)=\frac{4 l_{k, d}}{\pi R_{c}^{2}}\left[\cos ^{-1}\left(\frac{l_{k, d}}{2 R_{c}}\right)-\frac{l_{k, d}}{2 R_{c}}\left(1-\frac{l_{k, d}^{2}}{4 R_{c}^{2}}\right)^{\frac{1}{2}}\right] ; \\
& f_{R}\left(r_{i, d}\right)=\frac{4 r_{i, d}}{\pi R_{c}^{2}}\left[\cos ^{-1}\left(\frac{r_{i, d}}{2 R_{c}}\right)-\frac{r_{i, d}}{2 R_{c}}\left(1-\frac{r_{i, d}^{2}}{4 R_{c}^{2}}\right)^{\frac{1}{2}}\right] .
\end{aligned}
$$

Proof. See Appendix A for proof.

It should be noted that our hybrid system provides an increased probability of line-of-sight transmission for both the UEs as well as for the D2D links owing to the close proximity of APs and of devices. Therefore, we considered the special case of the free space path-loss exponent of $\alpha=2$.

Corollary 1: When making use of Theorem 1, which provides the general integral expression for the coverage probability at a typical D2D receiver, it becomes possible to derive an expression for the coverage probability for the special case of $\alpha=2$, yielding:

$$
C P=\int_{R_{1}}^{R_{2}} \mathcal{I}_{k, d}^{\frac{K}{N}-1} \mathcal{I}_{i, d}^{\frac{D}{N}-1} \frac{1}{R_{2}-R_{1}} \mathrm{~d} x
$$

where $\mathcal{I}_{k, d}$ and $\mathcal{I}_{i, d}$ are closed-form expressions for integral w.r.t. $l_{k, d}$ and $r_{i, d}$, respectively.

Proof. See Appendix B for proof.

Corollary 2: It is mathematically intractable to analyze (4) with respect to the number of RBs or UEs or D2Ds. To

\footnotetext{
$1 \frac{D}{N}$ and $\frac{K}{N}$ are also the normalized D2D-traffic load and the normalized user-traffic load respectively.
}

circumvent this problem, we make the following engineering approximation. If the distance between the transmitter and the receiver of the $\mathrm{D} 2 \mathrm{D}$ links, given by $x$, is a uniform random variable between $R_{1}$ and $R_{2}$ and the variation $R_{2}-R_{1}$ is extremely small compared to the cell radius $R_{C}$, the pdf (4) $f_{X}(x)$ of $x$ is concentrated more around the average value $\frac{R_{1}+R_{2}}{2}$. This is true for any typical D2D link in a cell of large radius. Hence, for such smaller range of Tx-Rx distances, the outermost integral, which is with respect to $x$, can be neglected in (4) and $x$ can be replaced by the average $\tilde{x}=\frac{R_{1}+R_{2}}{2}$. This implies that the approximate coverage probability for any $\alpha$ becomes:

$$
C P \approx\left[C P_{U E}\right]^{\frac{K}{N}-1}\left[C P_{D 2 D}\right]^{\frac{D}{N}-1}
$$

where $\quad C P_{U E}=\int_{0}^{2 R_{c}}\left(\frac{1}{1+\frac{T p_{k} l_{k, d}^{-\alpha}}{M p_{d} \tilde{x}-\alpha}}\right)^{M} f_{L}\left(l_{k, d}\right) \mathrm{d} l_{k, d} \quad$ and $C P_{D 2 D}=\int_{0}^{2 R_{c}} \frac{1}{1+\frac{T p_{d} r_{i, d}^{-\alpha}}{p_{d} \tilde{x}^{-\alpha}}} f_{R}\left(r_{i, d}\right) \mathrm{d} r_{i, d}$. For the case of $\alpha=2$, we get a closed form approximation with $C P_{U E}=\mathcal{I}_{k, d}$ and $C P_{D 2 D}=\mathcal{I}_{i, d}$

\section{B. Average Transmission Rate}

The ergodic rate of a TX-RX pair is given by [28],

$$
\begin{aligned}
R & =E\left[\log _{2}(1+S I R)\right] \\
& =\int_{t>0} P\left[\log _{2}(1+S I R)>t\right] \mathrm{d} t .
\end{aligned}
$$

Exploiting the fact that $\log _{2}(1+S I R)$ is a monotonically increasing function of SIR, we arrive at,

$$
R=\int_{t>0} P\left[S I R>2^{t}-1\right] \mathrm{d} t .
$$

Thus, the average rate is equivalent to the coverage probability evaluated at $T=2^{t}-1$ and then integrated over $t$. The coverage of a typical D2D link, which is at a distance of $l_{k, d}$ and $r_{i, d}$ from the $k^{t h}$ interfering AP and $i^{t h}$ interfering D2D link is given in Eq. (4). Thus, the average rate of the D2D link can be obtained by substituting $T=2^{t}-1$ into Eq. (4) and integrating the result over $t$, yielding:

$$
\begin{aligned}
R= & \int_{t=0}^{\infty} \int_{R_{1}}^{R_{2}}\left[\int_{0}^{2 R_{c}}\left(\frac{1}{1+\frac{\left(2^{t}-1\right) p_{k} l_{k, d}^{-\alpha}}{M p_{d} x^{-\alpha}}}\right)^{M} f_{L}\left(l_{k, d}\right) \mathrm{d} l_{k, d}\right]^{\frac{K}{N}-1} \times \\
& {\left[\int_{0}^{2 R_{c}} \frac{1}{1+\frac{\left(2^{t}-1\right) p_{d} r_{i, d}^{-\alpha}}{p_{d} x^{-\alpha}}} f_{R}\left(r_{i, d}\right) \mathrm{d} r_{i, d}\right]^{\frac{D}{N}-1} \frac{1}{R_{2}-R_{1}} \mathrm{~d} x \mathrm{~d} t . }
\end{aligned}
$$

\section{Simulation Results}

In this section, the performance of the coverage and average transmission rate of D2D links underlaying the DL of a DenseNet is studied. We consider a circular cell of radius $R_{c}$, 


\begin{tabular}{|l|r|}
\hline Parameter & Value \\
\hline Radius of Cell, $R_{c}$ & $500 \mathrm{~m}$ \\
\hline Number of APs, $L$ & 200 \\
\hline Number of UEs, $K$ & 100 \\
\hline Number of D2D links, $D$ & 100 \\
\hline Number of RBs, $N$ & 50 \\
\hline Number of Antennas at APs, $M$ & 8 \\
\hline Minimum Distance of D2D Pair, $R_{1}$ & $30 \mathrm{~m}$ \\
\hline Maximum Distance of D2D Pair, $R_{2}$ & $50 \mathrm{~m}$ \\
\hline Power budget of APs, $p_{c}^{\text {max }}$ & $30 \mathrm{dBm}$ \\
\hline Power budget of D2D links, $p_{d}^{\text {max }}$ & $20 \mathrm{dBm}$ \\
\hline Path-loss exponent, $\alpha$ & 3 \\
\hline \multicolumn{2}{|c|}{ Table II } \\
\hline
\end{tabular}

PARAMETERS USED FOR SIMULATIONS

where each of the $L$ uniformly distributed APs employs $M$ transmit antennas. All the $K$ UEs are distributed uniformly in the cell and share $N$ downlink RBs. These UEs form non-overlapping user-centric clusters with the closest AP. All the D D2D Rxs are uniformly distributed in the cell, while the D2D Txs are located at a distance $x$ from their respective receivers. This distance $x$ between a D2D pair is also uniformly distributed in $\left[R_{1}, R_{2}\right]$, while these D2D links reuse the underlying downlink RBs of UEs. Furthermore, all resource blocks are uniformly shared among the UEs and D2D links. In other words, if there are $K$ UEs or $D$ D2D links and $N$ resource blocks, then the RBs are uniformly distributed, leading to a RB reuse factor w.r.t UEs (or D2D links) of $\frac{K}{N}$ (or $\frac{D}{N}$ ) on an average. The power budget of the APs and D2D links is set to $p_{c}^{\max }$ and $p_{d}^{\max }$, as given in Table II, respectively. Random mapping of the D2D links to the UE's $\mathrm{RBs}$ is assumed. All the simulation results are generated by 1000 Monte Carlo runs using the parameters listed in Table II, unless otherwise specified.

The coverage probability of D2D links is depicted upon varying the number of available RBs in Fig. 2. First of all, it can be clearly seen that the analytical results closely match the simulations. As expected, upon increasing the target SIR, the coverage is decreasing owing to the severe interference experienced at the D2D Rx, resulting in its low SIR. It can be observed that upon reducing the number of available RBs, the D2D coverage is also reduced. This is because upon reducing the number of RBs, the number of UEs and D2D links reusing each RB is increased, which in turn means that there is an increase in the amount of interference experienced by the D2D $\mathrm{Rx}$, hence resulting in a reduced coverage probability for the D2D links. To analyze the decrease in coverage probability, the approximate coverage probability evaluated using Eq. (7) is also plotted. Since, $R_{1}=30 \mathrm{~m}$ and $R_{2}=50 \mathrm{~m}$ and the variation in the distance between $\mathrm{Tx}$ and $\mathrm{Rx}$ of $\mathrm{D} 2 \mathrm{D}$ is very small, the approximation is tight for all values of $T$. From the approximation plot, it can also be observed that, at any $T$, if the number of RBs $N$ decreases to $\frac{N}{2}$, then the coverage probability approximately decreases by a factor of $\left[C P_{U E}\right]^{\frac{K}{N}}\left[C P_{D 2 D}\right]^{\frac{D}{N}}$.

The impact of the number of D2D links as well as UEs on the coverage probability of D2D links is also analysed

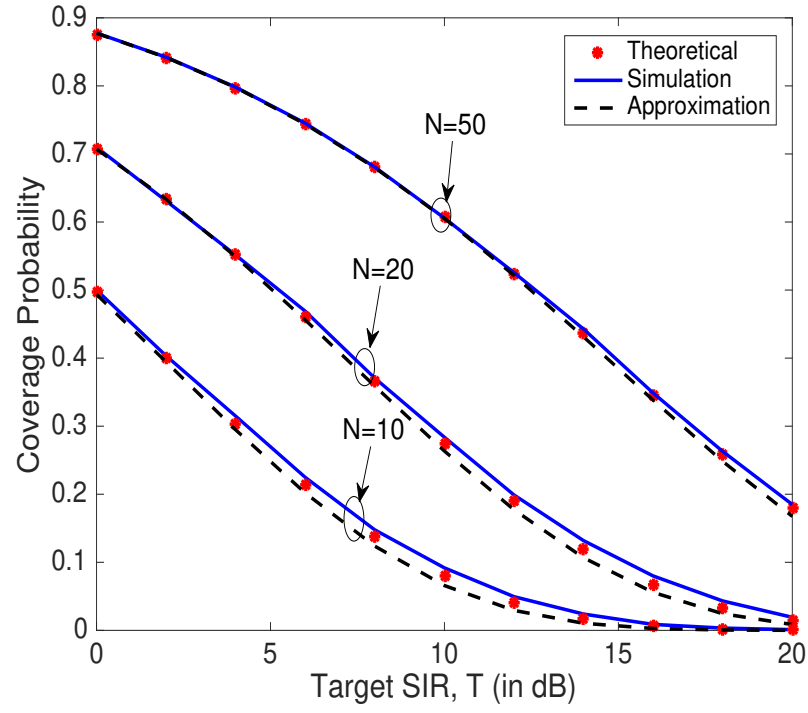

Figure 2. Coverage probability of the D2D links when the number of available RBs vary. All the system parameters are summarized in Table II and analytical results are obtained using Eq. (4).

in Fig. 3 and Fig. 4, respectively. It can be clearly observed from both figures that the D2D coverage is reduced upon increasing the number of D2D links or UEs. This is due to the fact that an increase in the number of D2D links or UEs implies that there is an increase in the number of interferers for a typical D2D link, which results in reducing the SIR of the D2D link and hence yielding a poorer coverage. If the number of D2D links increases from $D$ to $2 D$, then the coverage probability decreases by a factor of $\left[C P_{D 2 D}\right]^{\frac{D}{N}}$. Similarly, if the number of UEs increases from $K$ to $2 K$, then the coverage probability decreases by a factor of $\left[C P_{U E}\right]^{\frac{K}{N}}$. However, it is interesting to note that the coverage reduction of D2D links is more substantial upon increasing the number of UEs than upon increasing the number of D2D links. The reason behind this trend is the higher power budget of APs reserved for supporting their down-link transmission, thereby generating a higher amount of interference compared to the low power budget of D2D links. In other words, since $p_{k}>p_{d}$, $C P_{U E}<C P_{D 2 D}$.

In Fig. 5, the coverage probability of D2D links is presented as a function of the target SIR, T upon varying the number of transmit antennas at the APs. Again, our simulation results conform with our analytical results. Moreover, it is interesting to note that upon increasing the number of antennas at the APs, the reduction in coverage probability of D2D links shows a diminishing trend owing to the power normalization at APs.

In Fig. 6, the coverage probability of D2D links is presented as a function of the D2D reuse factor $D / N$, when the number of UEs varies from 100 to 300 . This figure corroborates our observation that as the ratio of $D / N$ doubles (in other words as the D2D-traffic load doubles), the coverage probability is reduced by a factor 


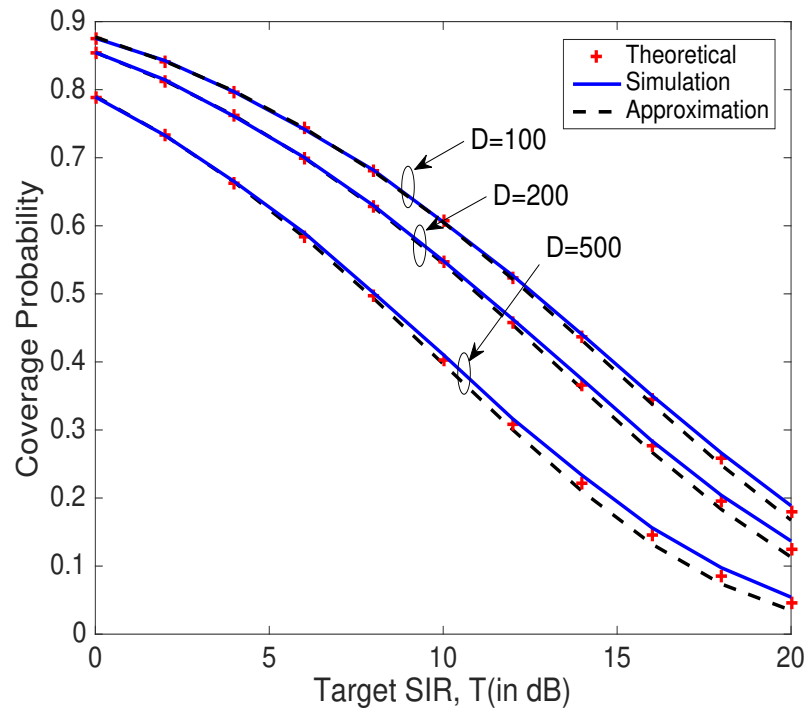

Figure 3. Coverage probability of the D2D links when the number of D2D links vary. All the system parameters are summarized in Table II and analytical results are obtained using Eq. (4).

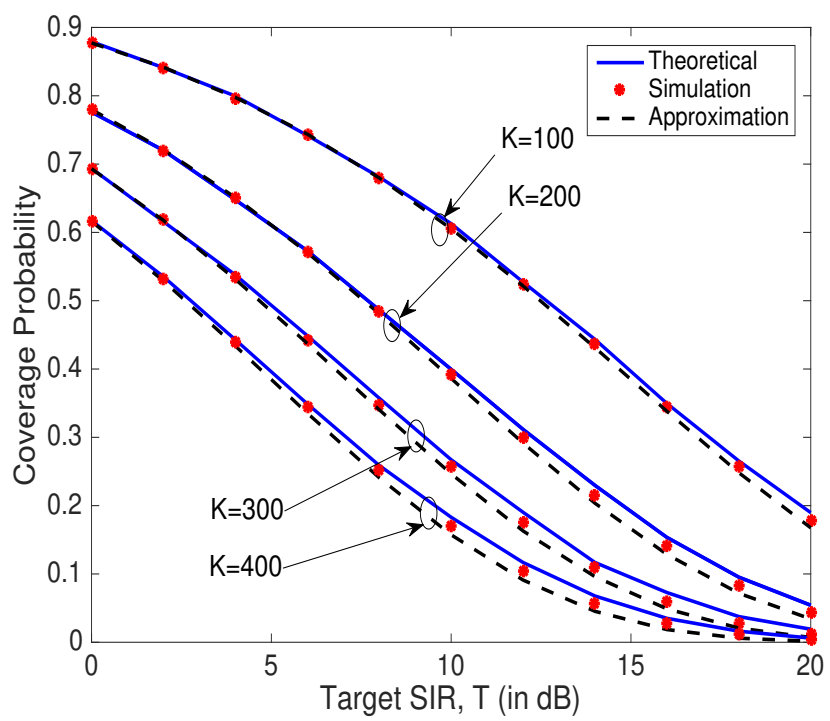

Figure 4. Coverage probability of the D2D links when the number of UEs vary. Here $L=800$, while all the other system parameters are summarized in Table II and analytical results are obtained using Eq. (4).

of about $\left[C P_{D 2 D}\right]^{\frac{D}{N}}$. Similarly, in Fig. 7 , the coverage probability of $D 2 D$ links isis portrayed vs. the reuse factor $K / N$, when the number of $\mathbf{D} 2 \mathrm{D}$ links is varied. Observe from the figure that the coverage probability is reduced by a factor of about $\left[C P_{U E}\right]^{\frac{R}{N}}$, when the normalized user-traffic load $K / N$ is doubled.

Fig. 8 depicts the coverage probability of D2D links for the special case of $\alpha=2$. The analytical results are generated using the simplified expression derived in Eq. (6). It can be clearly seen that the analytical results closely match the

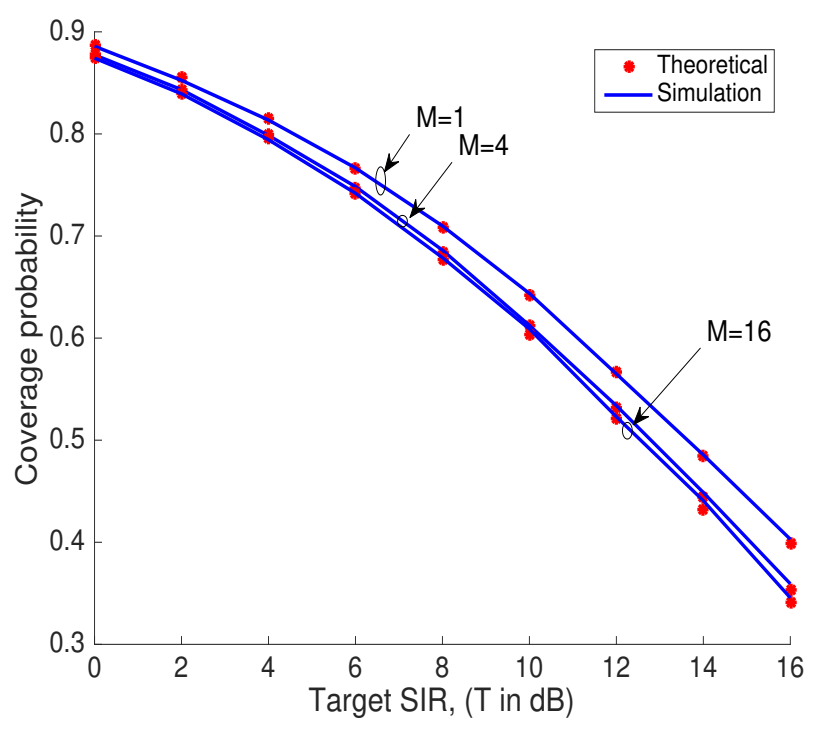

Figure 5. Coverage probability of the D2D links when the number of transmit antennas at APs vary. All the system parameters are summarized in Table II and analytical results are obtained using Eq. (4).

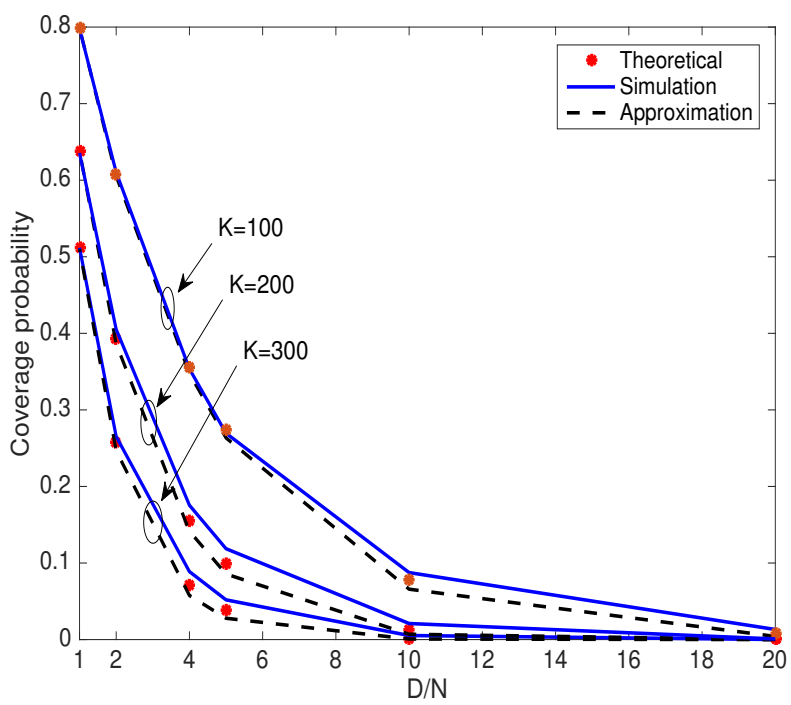

Figure 6. Coverage probability of the D2D links when the ratio $D / N$ varies. Here $L=300$, while all the system parameters are summarized in Table II and analytical results are obtained using Eq. (4).

simulations. Here we have a different combination of the number of D2D links in system and of the number of available RBs, where $D=100, N=50$ performs best, while $D=200, N=25$ performs worst. This is because when a large number of RBs is available, the number of UEs and D2D links transmitting in co-channel RBs decreases, hence reducing the overall interference at the D2D Rx, thereby improving both its SIR as well as its coverage. On the contrary, with more D2D links in system, the number of interferers increase resulting in a reduced coverage probability. 


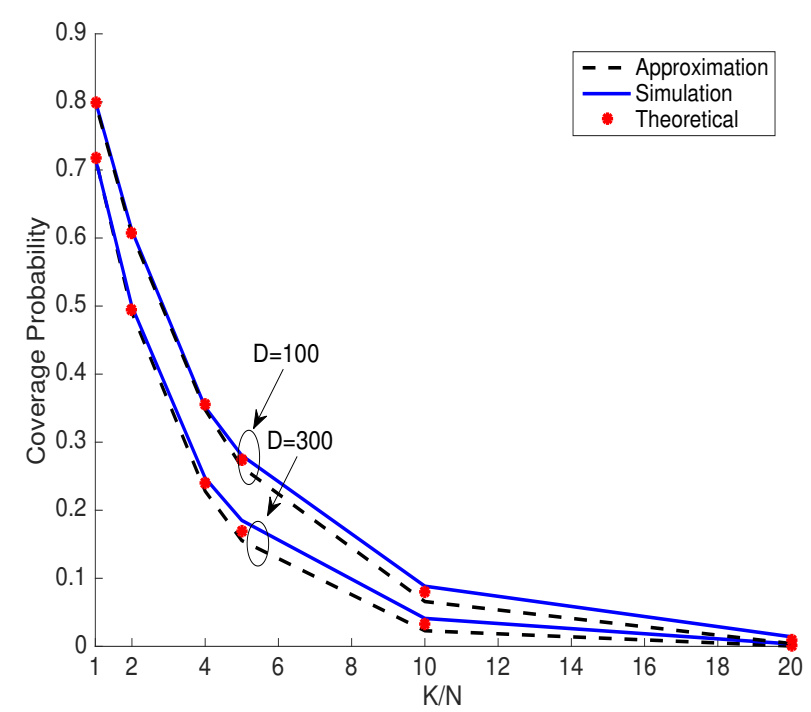

Figure 7. Coverage probability of the D2D links when the ratio $K / N$ varies. Here $L=300$, while all the system parameters are summarized in Table II and analytical results are obtained using Eq. (4).

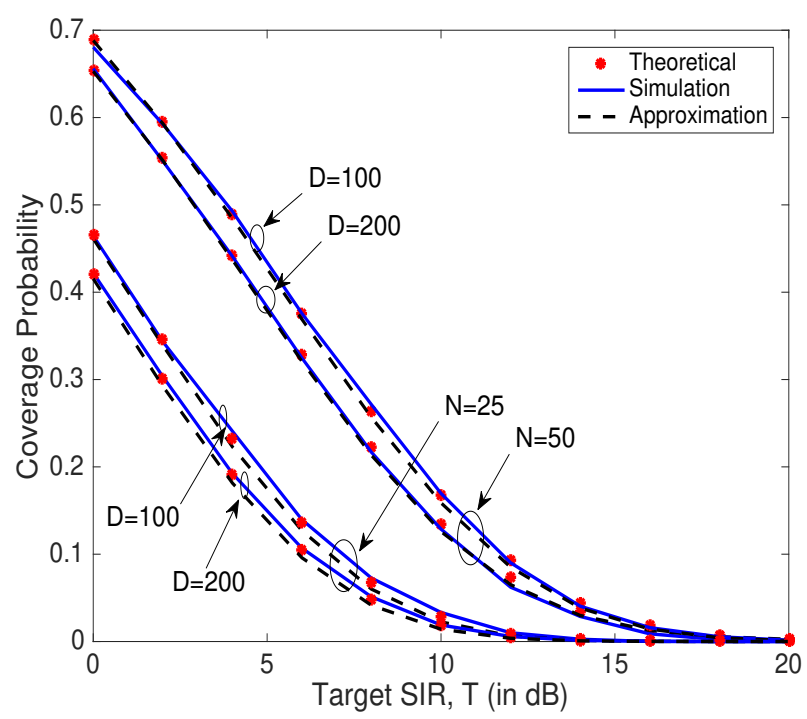

Figure 8. Coverage probability of the D2D links for the special case of $\alpha=2$. All the system parameters are summarized in Table II and analytical results are obtained using Eq. (6).

Finally in Fig. 9, the average transmission rate of D2D links is depicted as a function of the number of available RBs. Again, our analytical results conform with the simulations. It can be clearly observed that upon increasing the number of available RBs in the system, the average transmission rate of $\mathrm{D} 2 \mathrm{D}$ links is increased owing to the reduced reuse per RB. Moreover, upon increasing the number of antennas at the APs, the D2D transmission rate is reduced owing to the increased interference inflicted upon the $\mathrm{D} 2 \mathrm{D} \mathrm{Rx}$ due to multiple transmission streams being generated by the co- channel APs however the trend is diminishing owing to power normalization.

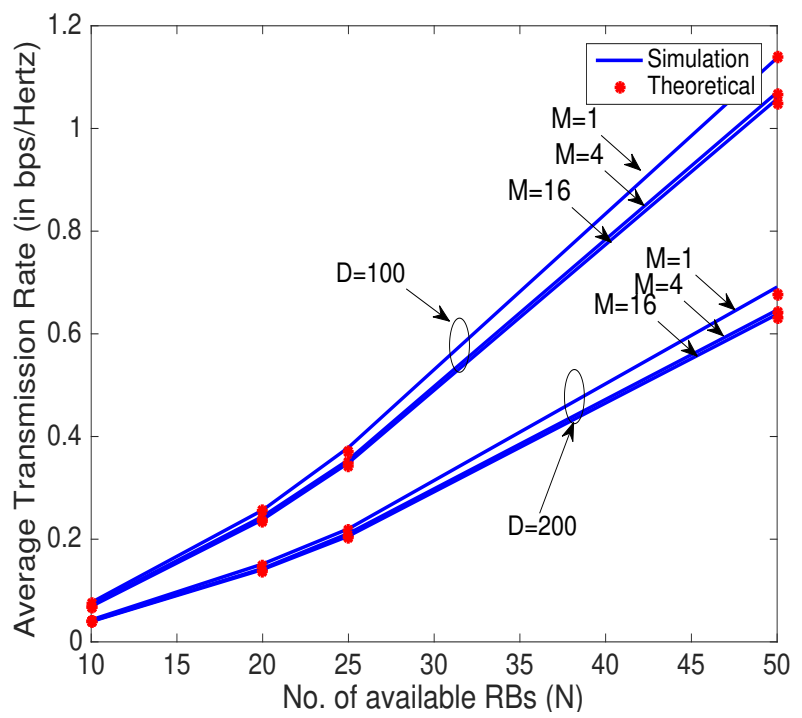

Figure 9. Average transmission rate of the D2D links when the number of available RBs vary. All the system parameters are summarized in Table II.

\section{CONCLUSIONS}

In this treatise, we proposed D2D communication underlaying the downlink of DenseNet, when the UEs form user-centric non-overlapping clusters with the closest AP. This integration resulted in a significantly modified interference scenario experienced both by the D2D links as well as by the UEs. We analytically derived both the coverage probability and the average transmission rate of D2D links under the proposed network model. Moreover, we also provided simplified analytical results for the coverage probability of D2D links for the case of $\alpha=2$. We observed from the performance results that our simulation results confirm our analysis. Moreover, the performance results also revealed that both the coverage probability and the transmission rate of D2D links are improved, when more orthogonal RBs are available in the system, as well as when the APs employ small the number of antennas. Finally, the impact of the D2D links on the coverage probability of UEs was also studied, which revealed that the UEs coverage is only modestly affected by the presence of D2D links, when numerous antennas are employed by the APs.

\section{APPENDIX A}

The coverage probability of a D2D link is given by

$$
\begin{aligned}
P\left[\beta_{d, n}>T\right] & =P\left[\frac{p_{d}\left|g_{d, d, n}\right|^{2} x^{-\alpha}}{I_{u d}+I_{d}}>T\right] \\
& =P\left[\left|g_{d, d, n}\right|^{2}>\frac{T\left(I_{u d}+I_{d}\right)}{p_{d} x^{-\alpha}}\right] .
\end{aligned}
$$


Since the fading gain of the D2D link is exponentially distributed, i.e., $g_{d, d, n} \sim \exp (1)$, we get,

$$
P\left[\beta_{d, n}>T\right]=E_{I_{u d}+I_{d}}\left[\exp \left(-\frac{T\left(I_{u d}+I_{d}\right)}{p_{d} x^{-\alpha}}\right)\right] .
$$

where we have

$$
\begin{aligned}
I_{d} & =\sum_{i \in \varphi_{d}} p_{d}\left|g_{i, d, n}\right|^{2} r_{i, d}^{-\alpha}, \\
I_{u d} & =\sum_{k \in \psi_{d}} \frac{p_{k}}{M} \sum_{j=1}^{M}\left|h_{k, d, n}^{j} w_{k, n}^{j}\right|^{2} l_{k, d}^{-\alpha}
\end{aligned}
$$

Substituting $I_{d}$ and $I_{u d}$ in Eq. (12), we obtain Eq. (14).

$$
\begin{array}{r}
P\left[\beta_{d, n}>T\right]=E_{I_{u d}+I_{d}}\left[\operatorname { e x p } \left(\frac{-T \sum_{k \in \psi_{d}} \frac{p_{k}}{M} \sum_{j=1}^{M}\left|h_{k, d, n}^{j} w_{k, n}^{j}\right|^{2} l_{k, d}^{-\alpha}}{p_{d} x^{-\alpha}}\right.\right. \\
\left.\left.+\frac{-T \sum_{i \in \varphi_{d}} p_{d}\left|g_{i, d, n}\right|^{2} r_{i, d}^{-\alpha}}{p_{d} x^{-\alpha}}\right)\right] .
\end{array}
$$

By exploiting the independence of $h_{i, d, n}$ and $h_{k, d, n}$, we can further simplify the above equation as

$$
\begin{array}{r}
P\left[\beta_{d, n}>T\right]= \\
\prod_{k \in \psi_{d}} \prod_{j=1}^{M} E\left[\exp \left(-\frac{\left.\left.T p_{k}\left|h_{k, d, n}^{j} w_{k, n}^{j}\right|^{2} l_{k, d}^{-\alpha}\right)\right]}{M p_{d} x^{-\alpha}}\right)\right] \times \\
\prod_{i \in \varphi_{d}} E\left[\exp \left(-\frac{\left.T p_{d}\left|g_{i, d, n}\right|^{2} r_{i, d}^{-\alpha}\right)}{p_{d} x^{-\alpha}}\right)\right] .
\end{array}
$$

The Moment Generating Function (MGF) of an exponentially distributed random variable $X$ with parameter $\lambda$ is given by $E[\exp (s X)]=\frac{\lambda}{\lambda-s}$ where $\lambda>s$. Since we have $\left|g_{i, d, n}\right|^{2} \sim$ $\exp (1)$ and $\left|h_{k, d, n}^{j} w_{k, n}^{j}\right|^{2} \sim \exp (1)$, we obtain

$$
P\left[\beta_{d, n}>T\right]=\prod_{k \in \psi_{d}} \prod_{j=1}^{M} \frac{1}{1+\frac{T p_{k} l_{k, d}^{-\alpha}}{M p_{d} x^{-\alpha}}} \prod_{i \in \varphi_{d}} \frac{1}{1+\frac{T p_{d} r_{i, d}^{-\alpha}}{p_{d} x^{-\alpha}}} .
$$

Therefore, the coverage probability of a typical D2D link can be formulated as:

$$
\begin{aligned}
& C P=\int_{0}^{2 R_{c}} \int_{0}^{2 R_{c}} \int_{R_{1}}^{R_{2}} P\left[\beta_{d, n}>T\right] f_{D}(d) \mathrm{d} x f_{L}\left(l_{k, d}\right) \mathrm{d} l_{k, d} f_{R}\left(r_{i, d}\right) \mathrm{d} r_{i, d} \\
&=\int_{R_{1}}^{R_{2}} \prod_{k \in \psi_{d}}\left[\int_{0}^{2 R_{c}}\left(\frac{1}{1+\frac{T p_{k} l_{k, d}^{-\alpha}}{p_{d} x^{-\alpha}}}\right)^{M} f_{L}\left(l_{k, d}\right) \mathrm{d} l_{k, d}\right] \times \\
& \prod_{i \in \varphi_{d}}\left[\int_{0}^{2 R_{c}} \frac{1}{1+\frac{T p_{d} r_{i, d}^{-\alpha}}{p_{d} x^{-\alpha}}} f_{R}\left(r_{i, d}\right) \mathrm{d} r_{i, d}\right] \frac{1}{R_{2}-R_{1}} \mathrm{~d} x \\
&= \int_{R_{1}}^{R_{2}}\left[\int_{0}^{2 R_{c}}\left(\frac{1}{1+\frac{T p_{k} l_{k, d}^{-\alpha}}{M p_{d} x^{-\alpha}}}\right)^{M} f_{L}\left(l_{k, d}\right) \mathrm{d} l_{k, d}\right]^{\frac{K}{N}-1} \times \\
& \times
\end{aligned}
$$

$$
\left[\int_{0}^{2 R_{c}} \frac{1}{1+\frac{T p_{d} r_{i, d}^{-\alpha}}{p_{d} x^{-\alpha}}} f_{R}\left(r_{i, d}\right) \mathrm{d} r_{i, d}\right]^{\frac{D}{N}-1} \frac{1}{R_{2}-R_{1}} \mathrm{~d} x
$$

The last equality is due to the fact that since the D2D and UEs are distributed uniformly, $\forall k \in \psi_{d}$, $\left[\int_{0}^{2 R_{c}}\left(\frac{1}{1+\frac{T p_{k} l_{k, d}^{-\alpha}}{p_{d} x^{-\alpha}}}\right)^{M} f_{L}\left(l_{k, d}\right) \mathrm{d} l_{k, d}\right]$ are equal and $\forall i \in \varphi_{d}$, $\left.\int_{0}^{2 R_{c}} \frac{1}{1+\frac{T p_{d} r_{i, d}^{-\alpha}}{p_{d} x^{-\alpha}}} f_{R}\left(r_{i, d}\right) \mathrm{d} r_{i, d}\right]$ are equal. Here, $\frac{D}{N}$ and $\frac{K}{N}$ are reuse factors of the D2D links and AP-UE links, which implies the number of interfering of D2Ds and UEs are $\frac{D}{N}-1$ and $\frac{K}{N}-1$ respectively.

\section{APPENDIX B}

In order to simplify the integral expression in Eq. (4), we introduce the shorthand of $r_{i, d}=2 R_{c} \cos \left(\theta_{i, d}\right)$. Then the integral w.r.t $r_{i, d}$ from Eq. (4) can be rewritten as:

$$
\begin{aligned}
& \mathcal{I}_{i, d}= \frac{16}{\pi} \int_{0}^{\frac{\pi}{2}} \frac{1}{1+a_{d}\left(\cos \left(\theta_{i, d}\right)\right)^{-2}} \times \\
&\left(\theta_{i, d} \cos \left(\theta_{i, d}\right) \sin \left(\theta_{i, d}\right)-\cos ^{2}\left(\theta_{i, d}\right) \sin ^{2}\left(\theta_{i, d}\right)\right) \mathrm{d} \theta_{i, d} \\
&= 1+2 a_{d} \ln (16)- \\
& 8\left(a_{d} \ln \left(1+\sqrt{1+\frac{1}{a_{d}}}\right)-\sqrt{a_{d}^{3}\left(1+a_{d}\right)}\right)+ \\
& 4 a_{d}\left(1+2 a_{d}\right),
\end{aligned}
$$

where $a_{d}=\frac{T d^{2}}{4 R_{c}^{2}}$. Similarly, the integral w.r.t $l_{k, d}$ from Eq. (4) when assuming $l_{k, d}=2 R_{c} \cos \left(\theta_{k, d}\right)$ can be rewritten as:

$$
\begin{aligned}
& \mathcal{I}_{k, d}= \frac{16}{\pi} \int_{0}^{\frac{\pi}{2}} \frac{1}{\left(1+b_{d}\left[\cos \left(\theta_{k, d}\right)\right]^{-2}\right)^{M}} \times \\
& {\left[\theta_{k, d} \cos \left(\theta_{k, d}\right) \sin \left(\theta_{k, d}\right)-\cos ^{2}\left(\theta_{k, d}\right) \sin ^{2}\left(\theta_{k, d}\right)\right] \mathrm{d} \theta_{k, d} } \\
&= \frac{16}{\pi} \int_{0}^{\frac{\pi}{2}} \frac{1}{\left(1+b_{d}\left[\cos \left(\theta_{k, d}\right)\right]^{-2}\right)^{M}} \times \\
& \frac{16}{\pi} \int_{0}^{\frac{\pi}{2}} \frac{\left[\theta_{k, d} \cos \left(\theta_{k, d}\right) \sin \left(\theta_{k, d}\right)\right] \mathrm{d} \theta_{k, d}-}{\left(1+b_{d}\left[\cos \left(\theta_{k, d}\right)\right]^{-2}\right)^{M}} \times \\
& {\left[\cos ^{2}\left(\theta_{k, d}\right) \sin ^{2}\left(\theta_{k, d}\right)\right] \mathrm{d} \theta_{k, d} } \\
&=\mathcal{I}_{k, d}^{(1)}-\mathcal{I}_{k, d}^{(2)}
\end{aligned}
$$

where $b_{d}=\frac{T p_{k} d^{2}}{4 M R_{c}^{2} p_{d}}$ and using Mathematica, we arrive at,

$$
\begin{array}{r}
\mathcal{I}_{k, d}^{(1)}=\frac{16}{\pi}\left[\frac { b _ { d } ^ { - M } \sqrt { \pi } \Gamma ( \frac { 3 } { 2 } + M ) } { 4 ( 1 + M ) ^ { 2 } \Gamma ( 1 + M ) } \left(( 1 + M ) _ { 2 } F _ { 1 } \left[M, \frac{3}{2}+M ; 2\right.\right.\right. \\
\left.+M ;-\frac{1}{b_{d}}\right] \\
-M_{P} F_{Q}\left[\left\{1+M, 1+M, \frac{3}{2}+M\right\},\{2+M, 2+M\},\right. \\
\left.\left.\left.-\frac{1}{b_{d}}\right]\right)\right],
\end{array}
$$




$$
\begin{array}{r}
\mathcal{I}_{k, d}^{(2)}=\frac{16}{\pi}\left[\frac { ( 1 + b _ { d } ) ^ { - M } \sqrt { \pi } } { 3 2 \Gamma ( M ) } \left(-4^{1-M}\left(1+b_{d}\right)^{M} e^{-i M \pi}(-1\right.\right. \\
\left.+4 b_{d} M+4 b_{d}^{2} M(1+M)\right) \Gamma\left(\frac{1}{2}-M\right) \Gamma(2 M) \\
+\frac{1}{b_{d}} \sqrt{1+\frac{1}{b_{d}}}(1+b) \Gamma\left(-\frac{3}{2}+M\right)\left(b_{d}(-3+2 M)(-15\right. \\
\left.+8 b_{d}^{2}(-1+2 M)+2 b_{d}(-13+7 M)\right) \\
+2 b_{d}(-2+M)\left(15-12 b_{d}(-2+M)\right. \\
\left.+4 b_{d}^{2}\left(2-3 M+M^{2}\right)\right)_{2} F_{1}\left[-\frac{1}{2}, 1 ; \frac{5}{2}-M ; 1+\frac{1}{b_{d}}\right] \\
-\left(15+b_{d}(26-14 M)+8 b_{d}^{3} M\left(-1+M^{2}\right)\right. \\
\left.\left.\left.\left.+4 b_{d}^{2}\left(2-5 M+3 M^{2}\right)\right)_{2} F_{1}\left[\frac{1}{2}, 1 ; \frac{5}{2}-M ; 1+\frac{1}{b_{d}}\right]\right)\right)\right]
\end{array}
$$

where ${ }_{2} F_{1}[$.$] and { }_{P} F_{Q}[$.$] are the Gauss hyper-geometric$ function and the generalized hyper-geometric function while $\Gamma($.$) is the gamma function [29].$

\section{REFERENCES}

[1] M. Kamel, W. Hamouda, and A. Youssef, "Ultra-dense networks: A survey," IEEE Communications Surveys Tutorials, vol. 18, no. 4, pp. 2522-2545, Fourthquarter 2016.

[2] A. Gupta and R. K. Jha, "A Survey of 5G Network: Architecture and Emerging Technologies," IEEE Access, vol. 3, pp. 1206-1232, 2015.

[3] H. Zhang, L. Song, Y. Li, and G. Y. Li, "Hypergraph theory: Applications in 5g heterogeneous ultra-dense networks," IEEE Communications Magazine, vol. 55, no. 12, pp. 70-76, Dec 2017.

[4] P. Janis, C. Yu, K. Doppler, C. Ribeiro, C. Wijting, K. Hugl, O. Tirkkonen, and V. Koivunen, "Device-to-device communication underlaying cellular communications systems," in International Journal on Communications, Network and System Sciences, vol. 2, June 2009, pp. $169-178$.

[5] D. Feng, L. Lu, Y. Yuan-Wu, G. Li, G. Feng, and S. Li, "Device-to-device communications underlaying cellular networks," IEEE Transactions on Communications, vol. 61, no. 8, pp. 35413551, August 2013.

[6] H. Sun, M. Wildemeersch, M. Sheng, and T. Q. S. Quek, "D2D enhanced heterogeneous cellular networks with dynamic TDD," IEEE Transactions on Wireless Communications, vol. 14, no. 8, pp. 4204 4218, Aug 2015.

[7] P. Phunchongharn, E. Hossain, and D. Kim, "Resource allocation for device-to-device communications underlaying LTE-advanced networks," IEEE Wireless Communications, vol. 20, no. 4, pp. 91-100, August 2013.

[8] A. Asheralieva and Y. Miyanaga, "QoS-Oriented Mode, Spectrum, and Power Allocation for D2D Communication Underlaying LTE-A Network," IEEE Transactions on Vehicular Technology, vol. 65, no. 12, pp. 9787-9800, Dec 2016.

[9] F. Malandrino, Z. Limani, C. Casetti, and C. F. Chiasserini, "Interference-aware downlink and uplink resource allocation in HetNets with D2D support," IEEE Transactions on Wireless Communications, vol. 14 , no. 5, pp. 2729-2741, May 2015.

[10] N. Cheng, H. Zhou, L. Lei, N. Zhang, Y. Zhou, X. Shen, and F. Bai, "Performance analysis of vehicular device-to-device underlay communication," IEEE Transactions on Vehicular Technology, vol. 66 , no. 6, pp. 5409-5421, June 2017.

[11] M. Salehi, A. Mohammadi, and M. Haenggi, "Analysis of d2d underlaid cellular networks: Sir meta distribution and mean local delay," IEEE Transactions on Communications, vol. 65, no. 7, pp. 2904-2916, July 2017.

[12] X. Lin, J. G. Andrews, and A. Ghosh, "Spectrum sharing for deviceto-device communication in cellular networks," IEEE Transactions on Wireless Communications, vol. 13, no. 12, pp. 6727-6740, Dec 2014.
[13] N. Lee, X. Lin, J. G. Andrews, and R. W. Heath, "Power control for d2d underlaid cellular networks: Modeling, algorithms, and analysis," IEEE Journal on Selected Areas in Communications, vol. 33, no. 1, pp. 1-13, Jan 2015.

[14] A. Sakr and E. Hossain, "Cognitive and energy harvesting-based d2d communication in cellular networks: Stochastic geometry modeling and analysis," IEEE Transactions on Communications, vol. 63, no. 5, pp. 1867-1880, May 2015.

[15] H. ElSawy, E. Hossain, and M. Alouini, "Analytical modeling of mode selection and power control for underlay $\mathrm{d} 2 \mathrm{~d}$ communication in cellular networks," IEEE Transactions on Communications, vol. 62, no. 11, pp. 4147-4161, Nov 2014.

[16] N. Bhushan, J. Li, D. Malladi, R. Gilmore, D. Brenner, A. Damnjanovic, R. T. Sukhavasi, C. Patel, and S. Geirhofer, "Network densification: the dominant theme for wireless evolution into 5G," IEEE Communications Magazine, vol. 52, no. 2, pp. 82-89, February 2014.

[17] J. Liu, M. Sheng, L. Liu, and J. Li, "Network densification in 5g: From the short-range communications perspective," IEEE Communications Magazine, vol. 55, no. 12, pp. 96-102, Dec 2017.

[18] — , "Effect of densification on cellular network performance with bounded pathloss model," IEEE Communications Letters, vol. 21, no. 2, pp. 346-349, Feb 2017.

[19] A. Shojaeifard, K. Wong, K. A. Hamdi, E. Alsusa, D. K. C. So, and J. Tang, "Stochastic geometric analysis of energy-efficient dense cellular networks," IEEE Access, vol. 5, pp. 455-469, 2017.

[20] M. Ding, P. Wang, D. López-Pérez, G. Mao, and Z. Lin, "Performance impact of los and nlos transmissions in dense cellular networks," IEEE Transactions on Wireless Communications, vol. 15, no. 3, pp. 2365-2380, March 2016.

[21] V. Garcia, Y. Zhou, and J. Shi, "Coordinated multipoint transmission in dense cellular networks with user-centric adaptive clustering," IEEE Transactions on Wireless Communications, vol. 13, no. 8, pp. 4297-4308, Aug 2014.

[22] Z. Zhang and R. Qingyang Hu, "Dense cellular network analysis with los/nlos propagation and bounded path loss model," IEEE Communications Letters, vol. 22, no. 11, pp. 2386-2389, Nov 2018.

[23] C. Ma, M. Ding, D. López-Pérez, Z. Lin, J. Li, and G. Mao, "Performance analysis of the idle mode capability in a dense heterogeneous cellular network," IEEE Transactions on Communications, vol. 66, no. 9, pp. 3959-3973, Sep. 2018.

[24] T. Ding, M. Ding, G. Mao, Z. Lin, D. López-Pérez, and A. Y. Zomaya, "Uplink performance analysis of dense cellular networks with los and nlos transmissions," IEEE Transactions on Wireless Communications, vol. 16, no. 4, pp. 2601-2613, April 2017.

[25] M. I. Poulakis, A. G. Gotsis, and A. Alexiou, "Multicell device-todevice communication: A spectrum-sharing and densification study," IEEE Vehicular Technology Magazine, vol. 13, no. 1, pp. 85-96, March 2018.

[26] X. Ge, S. Tu, G. Mao, C. X. Wang, and T. Han, "5G Ultra-Dense Cellular Networks," IEEE Wireless Communications, vol. 23, no. 1, pp. 72-79, February 2016.

[27] A. M. Mathai, An introduction to Geometrical Probability: Distributional Aspects with Applications. Montreal, Canada: Gordon and Breach Science Publishers, 1999.

[28] J. Andrews, F. Baccelli, and R. Ganti, "A Tractable Approach to Coverage and Rate in Cellular Networks," IEEE Transactions on Communications, vol. 59, no. 11, pp. 3122-3134, November 2011.

[29] M. Abramowitz and I. A. Stegun, Handbook of Mathematical Functions with Formulas, Graphs and Mathematical Tables. Dover Publications, Incorporated, 1974 